\title{
Radiation Shielding for Superconducting RF Cavity Test Facility at A0*
}

\author{
N. Dhanaraj, C. Ginsburg, I. Rakhno, G. Wu \\ Fermilab, P.O. Box 500, Batavia, IL 60510
}

December 10, 2008

\begin{abstract}
The results of Monte Carlo radiation shielding study performed with the MARS15 code for the vertical test facility at the A0 north cave enclosure at Fermilab are presented and discussed.
\end{abstract}

\section{Introduction}

The vertical test facility at the A0 north cave is planned to be used for testing $1.3 \mathrm{GHz}$ single-cell superconducting RF cavities with accelerating length of $0.115 \mathrm{~m}$. The operations will be focused on high accelerating gradients-up to $50 \mathrm{MV} / \mathrm{m}$. In such a case the facility can be a strong radiation source [1]. When performing a radiation shielding design for the facility one has to take into account gammas generated due to interactions of accelerated electrons with cavity walls and surroundings (for example, range of 3.7-MeV electrons in niobium is approximately $3.1 \mathrm{~mm}$ while the thickness of the niobium walls of such RF cavities is about $2.8 \mathrm{~mm}$ ). The electrons are usually the result of contamination in the cavity.

The radiation shielding study was performed with the MARS15 Monte Carlo code [2]. A realistic model of the source term has been used that describes spatial, energy and angular distributions of the field-emitted electrons inside the RF cavities. The results of the calculations are normalized using the existing experimental data on measured dose rate in the vicinity of such RF cavities.

\section{Geometry Model}

A plan view and cross sections of the developed three-dimensional model of the test facility are shown in Figs. 1 and 2. As for the color scheme employed to denote materials in the

\footnotetext{
*Work supported by Fermi Research Alliance, LLC, under contract DE-AC02-07CH11359 with the U. S. Department of Energy.
} 

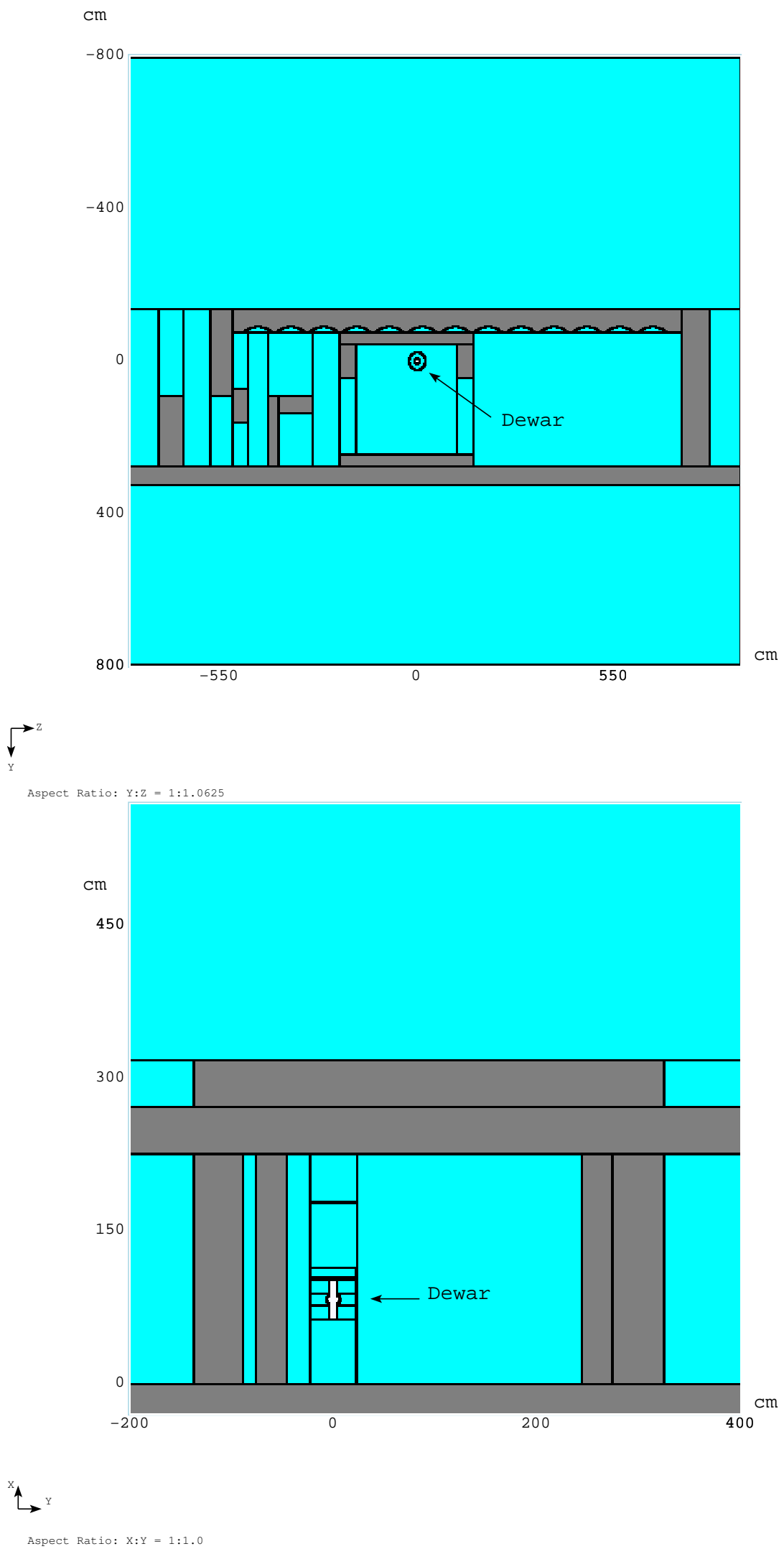

Figure 1: A plan view (top) and cross section (bottom) of the MARS15 model of the vertical test cryostat facility with a single-cell RF cavity inside the dewar. 

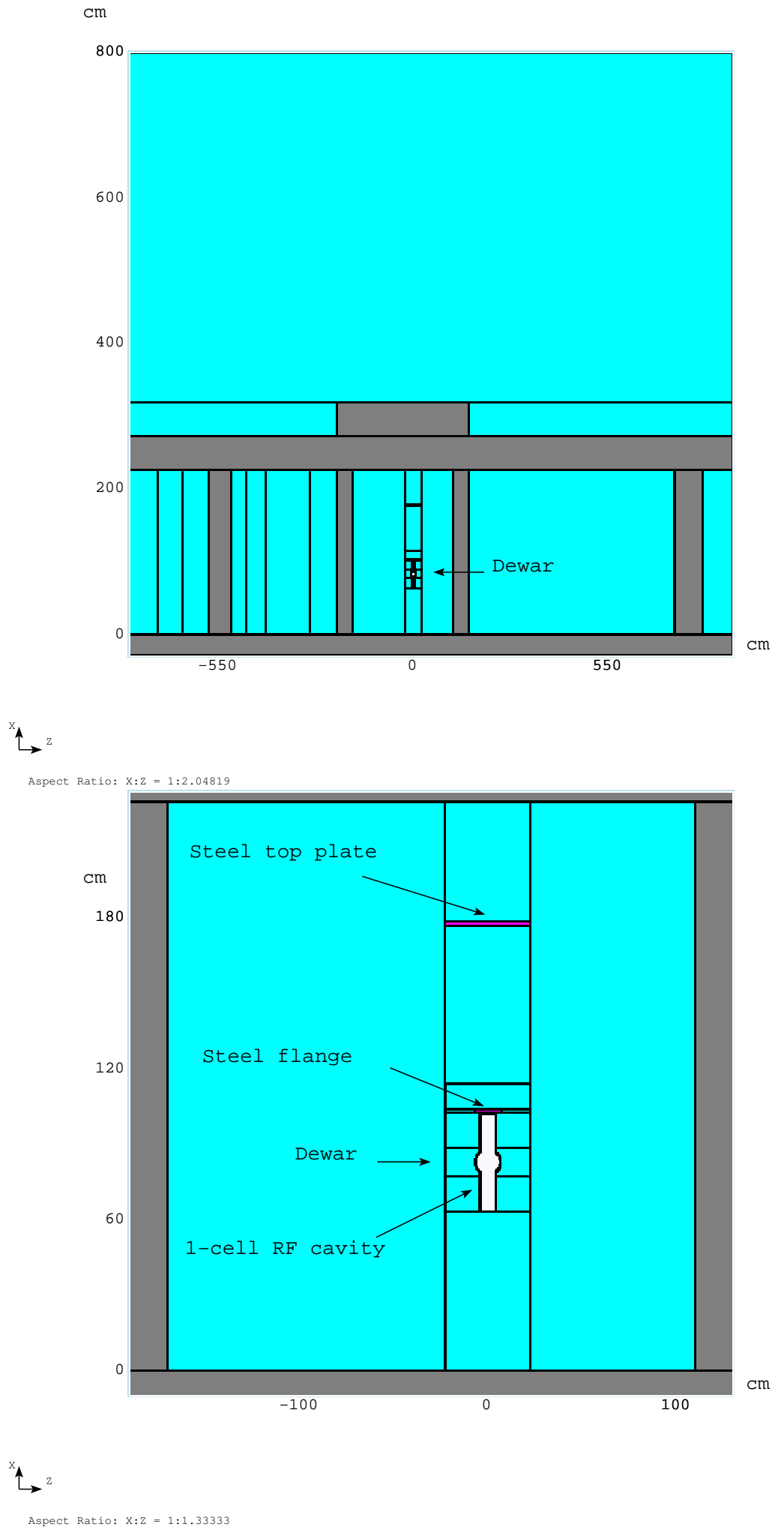

Figure 2: An elevation view of the MARS15 model of the vertical test cryostat facility with a single-cell RF cavity inside the dewar (top) and a fragment that shows also the $1.65-\mathrm{cm}$ steel flange and 1.9-cm steel top plate atop the dewar (bottom). 
model, the following convention applies: white, light blue, grey and pink colors correspond to vacuum, air, regular concrete and stainless steel, respectively. The boundaries between different regions are shown with black lines. It should be noted also that, when the resolution of a figure is inadequate to show small regions, these regions appear as black ones.

\section{Source term}

A realistic model has been developed recently in order to describe spatial, energy and angular distributions of field emission current generated in RF cavities at high accelerating gradients. The code FISHPACT [3] has been used to generate trajectories of electrons originating at the emitting site as shown in Fig. 3. The emitting site corresponds to location of the peak electric field in the cavity, so that the electrons emitted from this site have the highest probability of acquiring the maximum energy.

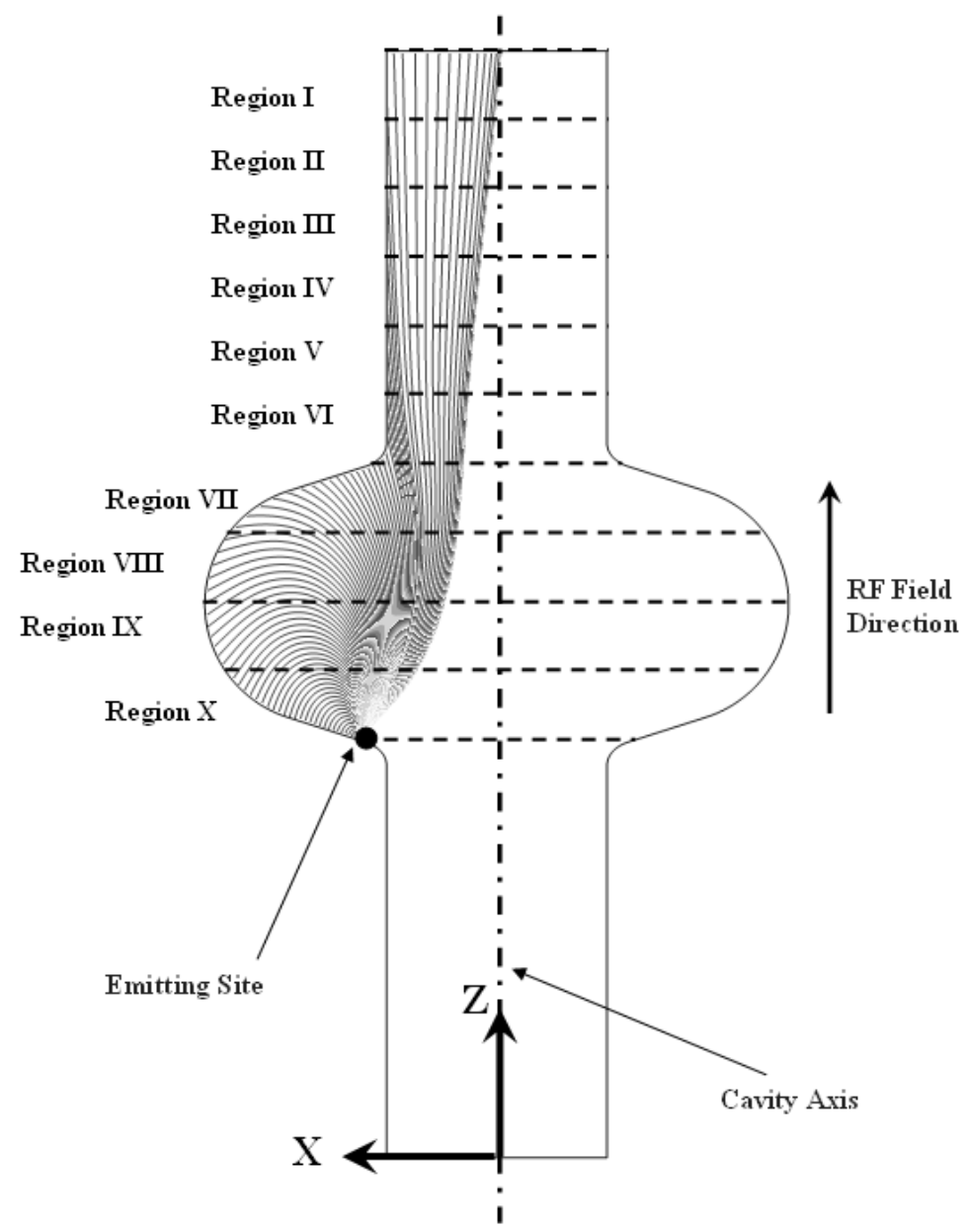

Figure 3: Sample electron tracks generated in a single-cell RF cavity with accelerating gradient of $42 \mathrm{MV} / \mathrm{m}$. The calculations were performed with the code described in Ref. [3]. 
The tracks shown in the Figure represent the paths of flight of the electrons for every 2 degree interval in the 180 degree space of the RF period. The tracks are symmetric around the vertical axis $Z$. The code also gives the final energy of an electron immediately before it strikes the inner wall of the cavity as well as angle of the incidence. The data, along with the maximum power available in the cavity, can be used to deduce the field emission current in the different spatial regions I through X as shown in Fig. 3.

The accelerating gradient of $42 \mathrm{MV} / \mathrm{m}$ was selected for the following reasons. First, the gradient in a cavity is limited by the superheating critical field, $H_{s h}$, which has a theoretical value of $230 \mathrm{mT}$ for niobium [4] and for which the corresponding gradient could be as high as $55 \mathrm{MV} / \mathrm{m}$. In our case we chose a widely measured $H_{s h}$ value of $180 \mathrm{mT}$ which corresponds to an accelerating gradient of $42 \mathrm{MV} / \mathrm{m}$. See, for example, Ref. [5] where measurements for alternative-shape single-cell cavities are described and the result is $175 \pm 15$ $\mathrm{mT}$. Second, for a fixed RF power, the higher the accelerating gradient, the lower field emission the cavity can tolerate. Any field emission takes a fraction of the energy stored in the cavity, so that the accelerating gradient drops. In other words, strong field emission and higher gradient such as $55 \mathrm{MV} / \mathrm{m}$ can not co-exist, while at $42 \mathrm{MV} / \mathrm{m}$ one can have some field emission loading. Therefore, generation of field emission current at $42 \mathrm{MV} / \mathrm{m}$ represents the worst case scenario from the standpoint of radiation shielding.

In our case the maximum electron energy corresponding to the accelerating gradient of $42 \mathrm{MV} / \mathrm{m}$ is $3.7 \mathrm{MeV}$ and it occurs in the region I. The total predicted field emission current for a given RF cycle is approximately $1.1 \mathrm{~mA}$ and this value seems to be a significant overestimate of a realistic value. The amount of electrons generated by a high electric field depends on the quality of the inner surface of the cavity and the quality is very difficult to describe numerically. On the other hand, the generated electron tracks are reliable because modeling of electron transport in a given electric and magnetic field is performed with a high accuracy. Therefore, to deduce the value of the field emission current, a comparison with a measured dose rate is used as described in the next section.

\section{Normalization of calculated dose}

The model of the field emission current described in the previous section lacks an essential component, that is the absolute value of the current. The value deduced from theoretical considerations [3] is extremely high (about $1.1 \mathrm{~mA}$ ) and not reliable and, therefore, can not be used for practical purposes. To that end, one uses experimental data obtained at DESY [6] for similar radiation tests of single-cell RF cavities.

Twelve years of experimental data for single-cell cavities from the DESY/TTF vertical test facility were analyzed, and are used to make realistic predictions. The X-ray dose rate was measured $5 \mathrm{~cm}$ off axis on top of the stainless-steel top plate (approximately $1 \mathrm{~m}$ to $1.5 \mathrm{~m}$ from the cavity), i.e., between the radiation shielding internal to the dewar and the shielding outside the dewar (the internal shielding used for those tests is shown in Refs. [7] and [8]). An analysis of the maximum x-ray dose rate, at maximum accelerating gradient, showed that the dose rate was less than $600 \mathrm{mrem} / \mathrm{hr} 90 \%$ of the time. The maximum $\mathrm{x}$-ray 
dose rate ever measured for such cavities was found to be $1500 \mathrm{mrem} / \mathrm{hr}$ (see Fig. 4).

A straightforward comparison of the dose measured in $90 \%$ of cases and calculated dose, other things being equal, gives rise to the conclusion that the field emission current is as low as $7.5 \mu \mathrm{A}$. Such an approach is a more reliable estimate of the field emission current generated in RF cavities when compared to theoretical predictions.

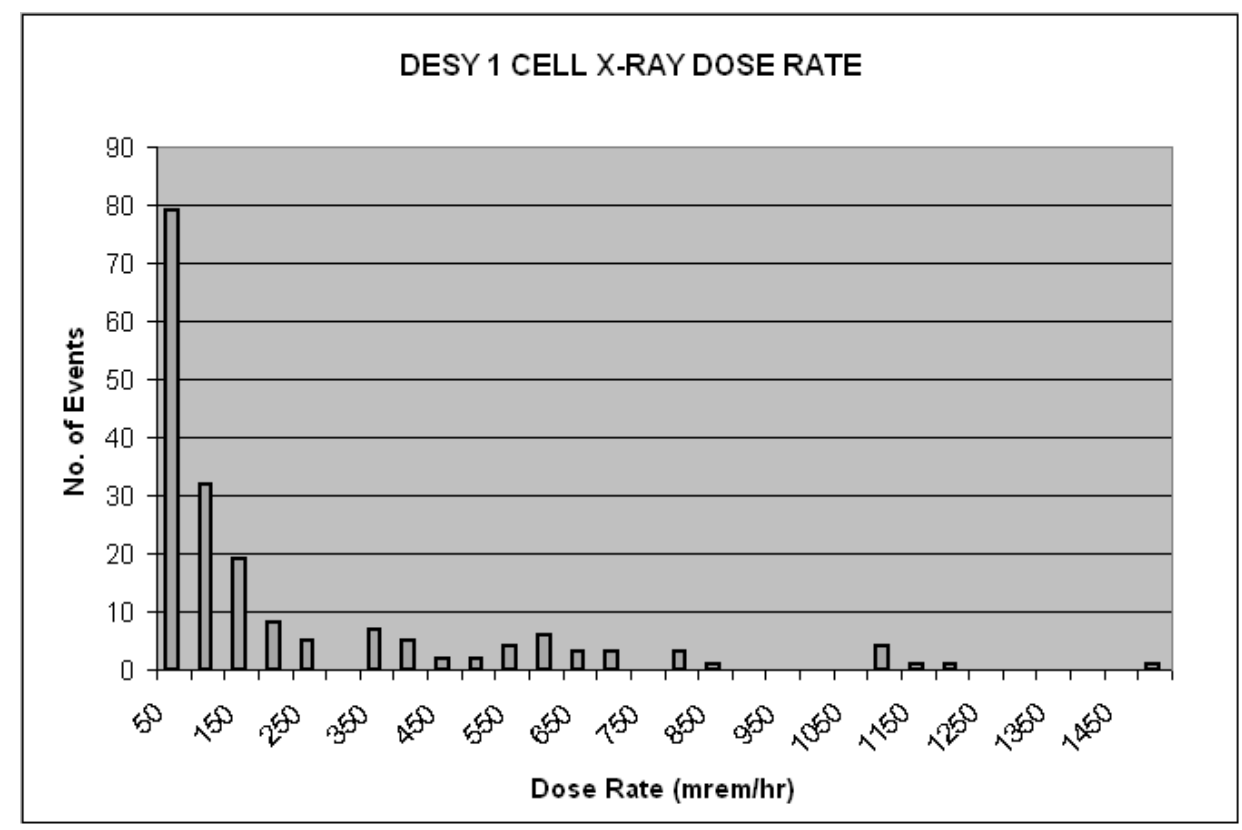

Figure 4: The distribution of measured dose rate at the DESY test facility for single-cell tests. The total number of recorded events with non-zero measured dose is equal to 186 .

\section{Calculated dose distributions around the facility}

The radiation shielding calculations have been performed for the facility without any extra internal shielding. The calculated distributions of the prompt dose around the facility are shown in Figs. 5 and 6 and in each case the dose was averaged over a layer as thick as 30 $\mathrm{cm}$. One can see from the Figures that a couple of hot spots reveal the dose slightly above $5 \mathrm{mrem} / \mathrm{hr}$ : (i) at the wall closest to the cavity, that is around $X=83 \mathrm{~cm}, Y=-130 \mathrm{~cm}, \mathrm{Z}=0$ $\mathrm{cm}$; (ii) on the roof above the cavity, that is around $X=320 \mathrm{~cm}, Y=Z=0 \mathrm{~cm}$. In both the cases the dose is about $15 \mathrm{mrem} / \mathrm{hr}$ and it can be reduced down to the acceptable level of $5 \mathrm{mrem} / \mathrm{hr}$ by means of an extra local shielding. A conservative estimate shows that the following thicknesses would suffice: (i) concrete- $10 \mathrm{~cm}$; (ii) steel $-4 \mathrm{~cm}$; (iii) lead $-2 \mathrm{~cm}$. 


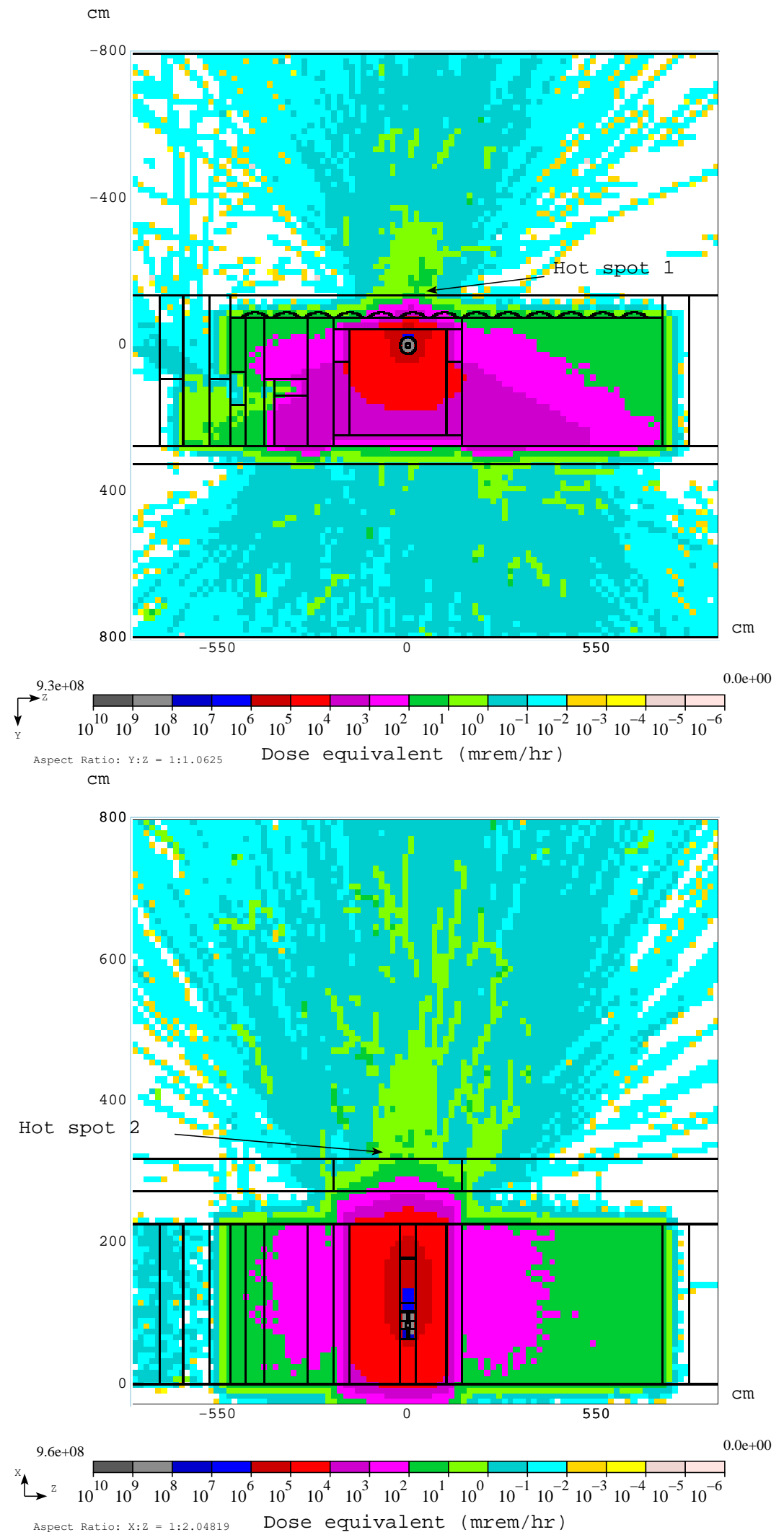

Figure 5: The calculated distributions of prompt dose rate around the test facility: a plan view (top) and an elevation view (bottom). 


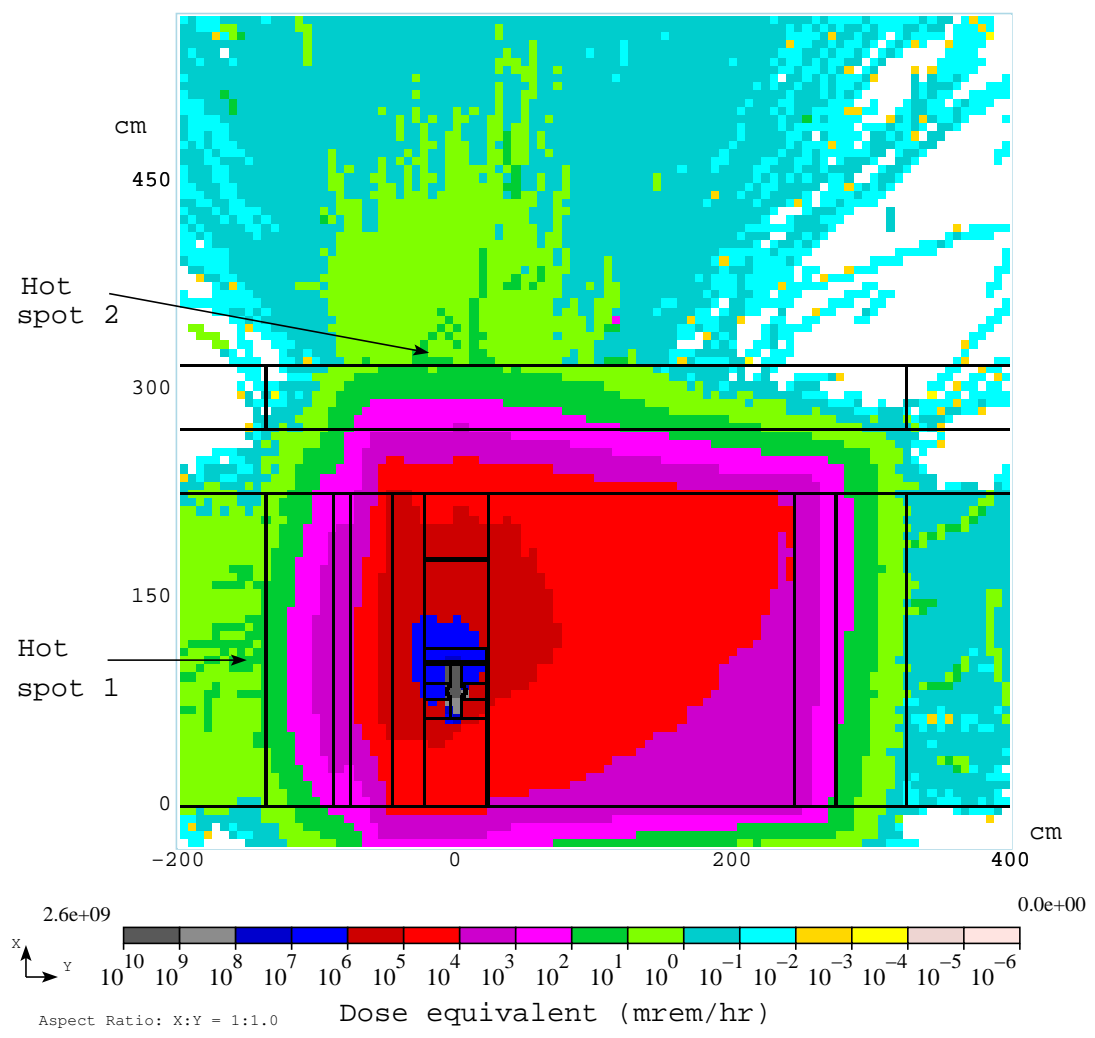

Figure 6: The calculated distribution of prompt dose rate around the test facility (a cross section).

With such an extra local shielding installed, the predicted dose outside the walls and above the roof of the facility will not exceed $5 \mathrm{mrem} / \mathrm{hr}$. At the same time, the regions more than $5 \mathrm{~m}$ away from the hot spots reveal the dose not exceeding $0.25 \mathrm{mrem} / \mathrm{hr}$.

\section{Conclusions}

The radiation shielding study performed with the MARS15 Monte Carlo code for the vertical test cryostat facility revealed that the existing shielding is adequate except for two hot spots. The dose at the two hot spots can be reduced by means of small amount of an extra local shielding. The predicted dose in regions farther than $5 \mathrm{~m}$ from the hot spots does not exceed $0.25 \mathrm{mrem} / \mathrm{hr}$.

\section{References}

[1] J. Knobloch, "Superconducting RF Cavity Test Facilities for Newman LaboratorySafety Report," Cornell University, Ithaca, NY (2001). 
[2] N.V. Mokhov, "The MARS code system userś guide version 13(95)," FermilabFN-628 (1995); N.V. Mokhov, S.I. Striganov, "MARS15 overview," Proc. Hadronic Shower Simulation Workshop, Batavia, Illinois, USA, 6-8 September, 2006, Vol. 896, pp. 50-60, American Institute of Physics, Melville, NY (2007); http://www-ap.fnal.gov/MARS/.

[3] E. Donoghue, G. Wu, J. Mammosser, R. Rimmer, M. Stirbert, L. Phillips and H. Wang, "Studies of electron activities in SNS-type superconducting RF cavities," Proc. SRF2005 Conference, Ithaca, New York, June 2005, TuP67.

[4] H. Padamsee, "The science and technology of superconducting cavities for accelerators,” Supercond. Sci. Technol., v. 14 (2001) pp. R28-R51.

[5] F. Furuta et al., "Experimental Comparison at KEK of High Gradient Performance of Different Single Cell Superconducting Cavity Designs," Proc. EPAC'06, Edinburgh, UK, June 2006.

[6] P. D. Gall and W.-D. Moeller, Private communication, DESY (2008).

[7] I. Rakhno, "Radiation Shielding Study for Superconducting RF Cavity Test Facility at Fermilab," Fermilab-TM-2350-AD (2006).

[8] I. Rakhno, "Radiation Shielding Issues for Superconducting RF Cavity Test Facility at Fermilab," Fermilab-TM-2367-AD (2006). 